\title{
Offen, verantwortlich und verantwortlich offen
}

\author{
Zum Verhältnis von RRI und Open Science
}

\author{
Johann Jakob Häußermann, Fraunhofer Center for Responsible Research and Innovation am IAO, Hardenbergstr. 20, 10623 Berlin \\ (johann-jakob.haeussermann@iao.fraunhofer.de) \\ Marie Heidingsfelder, Fraunhofer Center for Responsible Research and Innovation am IAO, \\ (marie-lena.heidingsfelder@iao.fraunhofer.de)
}

Sowohl Open Science als auch Responsible Research and Innovation (RRI) zielen auf tiefgreifende Transformationen im Innovationssystem durch eine stärkere Öffnung von Forschung und Entwicklung für gesellschaftliche Akteure. Trotz dieser grundsätzlichen Nähe beider Konzepte wurde ihr Verhältnis bisher nicht eingehend analysiert. Dieser Beitrag möchte einen ersten Ansatz bieten, die Ähnlichkeiten und Unterschiede von RRI und Open Science hinsichtlich des diskursiven Ursprungs der beiden Metakonzepte, ihrer jeweiligen Herausforderungen und Ziele sowie ihrer Akteure und der Rolle der Gesellschaft zu diskutieren. Darauf aufbauend werden Implikationen und Potenziale einer konzeptionellen Integration aufgezeigt. Der Artikel schließt mit einem Ausblick auf mögliche Ansätze einer Integration.

\section{Open, responsible, and responsibly open}

On the relationship between $R R I$ and Open Science

Both Open Science and Responsible Research and Innovation aim to initiate profound transformations of innovation systems by increased integration of societal actors in research and development. Despite this common ground, the relationship between both concepts so far has not been addressed and mutually analyzed. This contribution offers a first approach to discussing both overlaps and differences concerning the discursive origins of both meta-concepts, their respective challenges and aims as well as their agents and the role of society. We derive implications and highlight the potential of integrating the two concepts. The article concludes with an outlook on implications for further research.

KEYWORDS: open science, responsible innovation, open access, research policy

This is an article distributed under the terms of the Creative Commons Attribution License CCBY 4.0 (https://creativecommons.org/licenses/by/4.0/)

https://doi.org/10.14512/tatup.26.1-2.31

Eingereicht: 15. 03.2017. Angenommen: 17.05.2017

\section{Einleitung}

Mit der Ernennung von Carlos Moedas als EU-Kommissar für Forschung, Wissenschaft und Innovation hat Open Science als forschungspolitisches und forschungsstrategisches Thema deutlich an Relevanz und Aufmerksamkeit gewonnen: Angetrieben durch die technologischen Entwicklungen der Digitalisierung und des Internets soll Open Science die Prozesse und Ergebnisse der Wissensproduktion für die Gesellschaft öffnen und damit das Wissenschaftssystem in einer second scientific revolution (Bartling und Friesike 2014) grundlegend transformieren (Moedas 2015; European Commission 2016).

Mit dem ähnlichen Ziel, Forschungs- und Innovationsprozesse mit der und für die Gesellschaft zu ermöglichen (Owen et al. 2012), kann auf forschungsstrategischer Ebene der Europäischen Kommission die zunehmende Bedeutung und Ausdifferenzierung eines zweiten Konzeptes beobachtet werden: Responsible Research and Innovation (RRI). Beide Konzepte - Open Science und RRI - zielen auf Transformationen im Innovationssystem, die Prozesse der Wissensproduktion, ebenso wie Prozesse der Wissensdissemination und des Wissenstransfers betreffen.

Vor dem Hintergrund dieser grundlegenden Gemeinsamkeit versucht dieser Beitrag, die Schnittmengen beider Metakonzepte sichtbar zu machen und Potenziale für eine gegenseitige Bereicherung aufzuzeigen. Das Ziel ist dabei weniger eine theoretische Flurbereinigung, als vielmehr eine praxisorientierte Darstellung, ob und wie Methoden und Forschungsinteressen beider Ansätze im jeweils anderen Bereich genutzt werden können. Ziel des Artikels ist daher nicht eine umfassende konzeptionelle Integration von Open Science und RRI, sondern ein erster Brückenschlag zwischen beiden Metakonzepten, und ein Plädoyer, Open Science als Teil verantwortlicher Forschung und Innovation zu verstehen. Zur Argumentation der These, dass RRI und Open Science über vielfältige Verbindungslinien verfügen, werden zunächst wesentliche Aspekte beider Ansätze dargestellt. Im Folgenden werden Schnittmengen und Unterschiede zwischen 
beiden beschrieben, und es wird anschließend diskutiert, welche Potenziale sich daraus jeweils gewinnen lassen. Da das Verhältnis beider Metakonzepte Implikationen birgt, die im Rahmen dieses kurzen Beitrags nicht umfassend erfasst werden können, schließt der Artikel mit einem Ausblick auf mögliche Ansätze einer Integration.

\section{Hintergrund}

\section{RRI}

Obgleich das Aufkommen von Responsible Research and Innovation zeitlich einigermaßen genau datiert werden kann und der Diskurs spätestens seit 2011 deutlich an Kontur gewonnen hat (Tancoigne et al. 2016), entspricht RRI weiterhin einem noch nicht endgültig stabilisierten und offenen Netz von Auffassungen und Perspektiven (Rip 2016). Hinter diesen liegen unterschiedliche Einflusslinien, die RRI hervorgebracht haben. Besondere Bedeutung kommt dabei Fragen im Kontext der Nanotechnologie in den frühen 2000er-Jahren zu, die zu Überlegungen zu verantwortlicher Forschung und Entwicklung führten (Barben et al. 2007; Rip 2010). Darüber hinaus können weitere (disziplinäre) Beiträge aus Bereichen der Verantwortungsethik, Wissenschaftsethik, der Technology-Governance-Theorie, der Innovationswissenschaft, der Science and Technology Studies, des Social Shaping Approach of Technology, der ELSI/ELSAAnsätze, des Value Sensitive Designs und der Technikfolgenabschätzung (TA) identifiziert werden, die zu einem umfassenden und vielschichtigen Diskurs um RRI geführt haben. Während RRI also unterschiedliche Perspektiven und Entwicklungen unter einem selbst offeneren Begriff verbindet, stellt es zugleich einen Ort für weitere Entwicklungen und Ansätze verschiedener Akteure zur Verfügung.
RRI geht jedoch insofern über Entwicklungen der TA hinaus, als die Perspektive von einzelnen Entscheidungen und Technologien auf das grundlegende Verhältnis von Technologie und Gesellschaft in Innovations- und Forschungsprozessen verschoben wird (Schomberg 2013). Im Vordergrund steht also weniger die Identifikation negativer Neben- oder Sekundärfolgen, als die Frage, wie das institutionelle Verhältnis und die Arbeitsteilung der unterschiedlichen Akteure innerhalb eines Innovationssystems (Wissenschaft, Wirtschaft, Politik, Gesellschaft) zu organisieren ist und welche Innovationen überhaupt gesellschaftlich gewollt werden (können). Indem RRI Verantwortung zum Kernbegriff macht, rückt es die normative Ausrichtung von Forschung und Innovation in den Fokus und fragt nach den ,richtigen“ Zwecken und Zielen von Innovation, Forschung und Wissenschaft (Schomberg 2014) oder fokussiert auf deren Prozesse (Stilgoe et al. 2013).

Die Frage nach der Verantwortung von WissenschaflerInnen und der Verantwortbarkeit von Forschungs- und Innovationsprozessen verweist auf den Bedarf der Legitimierung und einer sozialen Lizenz sowohl für öffentliche Institutionen als auch für privatwirtschaftliche Unternehmen (Rip 2014). Damit trägt RRI zu einer Koverantwortung der moralischen Arbeit (moral labour) zwischen allen Akteuren des Innovationssystems bei (Owen et al. 2013). Diese institutionelle Transformation bedeutet nicht zuletzt auch die Veränderung von Rollenbildern einzelner Akteure, neue Anforderungen an Unternehmen, Forschungsorganisationen und die Politik, bis hin zu neuen Rollen für Kunden, Laien und BürgerInnen (Rip 2016). In diesem Sinn kann RRI selbst als eine andauernde soziale Innovation verstanden werden, deren Vision für eine institutionelle Transformation im Sinne eines adaptiven und responsiven Verhältnisses zwischen den beteiligten Akteuren offen für unterschiedliche Perspektiven und Umsetzungen ist (Rip 2014; Schomberg 2013). Indem

\section{RRI zielt auf eine grundlegende institutionelle Transformation des Innovationssytems und rückt deren normative Ausrichtung in den Fokus.}

Insbesondere die TA, die sich aufgrund des steigenden Bewusstseins für unerwünschte Neben- oder Sekundärfolgen technisch-wissenschaftlicher Entwicklungen in der zweiten Hälfte des zwanzigsten Jahrhunderts zum zentralen Bestandteil von Forschungs- und Entwicklungsprogrammen entwickelte, ist für ein konzeptuelles und praxisorientiertes Verständnis von RRI maßgeblich (Fisher und Rip 2013; Grunwald 2014). Besonders relevant sind dabei die partizipative (p)TA, die auf den Einbezug gesellschaftlicher Gruppen und Stakeholder in die Technologieentwicklung zielt, sowie die konstruktive (k)TA, die eine grundlegende Integration von gesellschaftlichen Aspekten und Perspektiven in Forschungs- und Innovationsprozesse anstrebt.
RRI Verantwortung und soziale Rechenschaft einfordert, kann es sogar als Beitrag zu einer offensiven „Ethisierung der Technik" verstanden werden (Bogner 2013; Bogner et al. 2015).

Ungeachtet seiner terminologischen Offenheit und sogar expliziter Unklarheit innerhalb der Kommission (Rip 2016) konnte RRI insbesondere im Rahmen von Horizon 2020 einige Wirkmächtigkeit entfalten. Als Science with and for Society (SwafS) ist RRI integraler Bestandteil der europäischen Förderpolitik und schafft sich damit gewissermaßen eigene Realitäten (Bogner et al. 2015) und nicht zuletzt auch Abhängigkeiten und Interessen, so dass ein reflexiver Umgang mit normativen Ansprüchen, Interpretationen und Umsetzungen notwendig ist. 


\section{Open Science}

Auch Open Science kann auf etablierte Theorien und Ansätze zurückgeführt werden, insbesondere auf die Beschreibung und Untersuchung neuer Wissensformen und -produktionsweisen sowie auf Ansätze, in denen die Zugänglichkeit zu wissenschaftlichen Erkenntnisprozessen und Ergebnissen als Pfeiler der deliberativen Demokratie gesehen wird (Elam und Bertilsson 2003). Beide Argumentationslinien werden im Folgenden kurz dargestellt.

Aufgrund der zunehmenden Komplexität von Akteuren und Beziehungen im Innovationssystem (Etzkowitz und Leydesdorff 2000; Carayannis und Campbell 2009, 2011), werden seit etwa freien Zugang zu wissenschaftlichen Daten (Open Data), Evaluationssystemen (Open Science Evaluation) und Umgebungen, in denen experimentelle Forschungen wiederholt werden können (Open Reproducible Research) (Schmidt et al. 2016). Darüber hinaus beinhaltet Open Science auch Ansätze, in denen ,nicht-professionelle Wissenschaftler/Innen“ in wissenschaftliche Erkenntnisprozesse einbezogen werden (Hand 2010; Powell und Colin 2009), wie beispielsweise Bürgerwissenschaften oder partizipative Forschung. Zusammenfassend kann Open Science damit als Ansatz und Anspruch verstanden werden, wissenschaftliche Erkenntnis- und Verbreitungsprozesse umfassend für die Gesellschaft offen zu legen und zur Verfügung zu stellen.

\section{Open Science und Citizen Science zielen auf tiefgreifende Transformationen der Prozesse von Wissensproduktion, Wissensdefinition und Wissenstransfer.}

drei Dekaden neue Wissensformen und -produktionsweisen beschrieben, die stärker auf die jeweiligen gesellschaftlichen Praktiken, Voraussetzungen und Herausforderungen eingehen bzw. in einem engeren, inhärenteren Austausch und Verhältnis mit eben diesen stehen (Funtowicz und Ravetz 1993; Gibbons et al. 1994; Gibbons 2000; Nowotny et al. 2003; Carayannis und Campbell 2009). Die Formen dieses post-normalen, Mode-2- oder Mode-3Wissens verweisen auf die dynamischen Mechanismen und Strukturen von Wissensproduktion und -dissemination in modernen, von vielen Akteuren geprägten und ko-evolutiven Innovationssystemen.

Mit dem democratic turn hat sich, so die zweite Grundlage für die aktuelle Diskussion um Open Science, das Selbstverständnis der Wissenschaft im Verhältnis zur Gesellschaft gewandelt: Galt noch bis in die 1980er-Jahre das Prinzip einer unidirektionalen Kommunikation von der Wissenschaft in die Gesellschaft, bei der es vor allem darum ging, das Verständnis und die Akzeptanz für wissenschaftliche Forschung bei „der“ Öffentlichkeit durch möglichst gute Information zu sichern, öffnete der democratic turn den Blick von einem ,public understandment of science“ hin zu einem ,public engagement in science“ (Siune et al. 2009; McCallie et al. 2009; Schäfer 2009). Auf diese Weise wurde der defizitäre Blick auf die Gesellschaft - ,people are empty, imperfect vessels waiting to be filled with good information" (Siune et al. 2009, S. 51) - zugunsten einer Perspektive aufgegeben, in der gesellschaftliche Akteure über relevante und wertvolle Wissensbestände verfügen, die sie in einen Dia$\log$ mit WissenschaftlerInnen einbringen können.

Gleichzeitig bieten moderne Informations- und Kommunikationstechnologien eine Infrastruktur, in der wissenschaftliche Daten, Erkenntnisse und Publikationen breit zugänglich gemacht werden können. Open Science geht aber über den freien $\mathrm{Zu}$ gang zu Ergebnissen (Open Access) hinaus und umfasst auch den
Wie Fecher und Friesike (2014) verdeutlichen, liegen unter dieser übergreifenden Definition unterschiedliche Ansätze für Open Science vor, die für signifikante Differenzen in der Schwerpunksetzung und Gestaltung des Konzepts stehen. Die von ihnen dargestellten Denkschulen umfassen dabei sowohl Ansätze, die das demokratische Recht auf die Zugänglichkeit wissenschaftlicher Ergebnisse durch BürgerInnen betonen, als auch eher technische Ansätze, die sich unter der Überschrift Open Science mit Fragen der digitalen Infrastruktur oder den Evaluationssystemen für Wissenschaft auseinandersetzen.

\section{Schnittmengen und Unterschiede}

Wie die Darstellung beider Konzepte zeigt, haben RRI und Open Science auf unterschiedlichen Ebenen Ähnlichkeiten: Beide zielen auf tiefgreifende Transformationen der Prozesse von Wissensproduktion, Wissensdefinition und Wissenstransfer; beide sind forschungspolitisch initiiert und beide haben ihrer Neuheit zum Trotz feste Wurzeln in bestehenden Ansätzen und Theorien. Darüber hinaus ist Open Access, das heißt der freie Zugang zu den Ergebnissen von Forschung und Innovation, ein maßgeblicher Pfeiler innerhalb von RRI (European Commission 2012; European Commission 2013). Dieser Bezug verweist auf einen möglichen und vielleicht sogar notwendigen Brückenschlag zwischen beiden Konzepten. Dies soll im Folgenden anhand einer ersten Gegenüberstellung besonders relevanter Schnittmengen und Unterschiede versucht werden.

- Herkunft und Stand der Debatte: Beide Metakonzepte sind forschungspolitisch initiiert, das heißt die Einbindung in politische Programme und Förderkonzepte trägt entscheidend zu ihrer Entstehung und Wirkung bei. Zudem sind beide dyna- 
mische und offene Konzepte, insofern Inhalt, Ziel und intendierte Effekte nicht endgültig definiert und ,abgeschlossen“ sind. RRI und Open Science unterscheiden sich jedoch im Hinblick auf ihre konkreten Hintergründe und konzeptuellen Einflüsse. Während RRI unter anderem im Kontext von Diskussionen um verantwortliche Forschung und Entwicklung, Technikfolgenabschätzung und im Rahmen der Science and Technology Studies entstanden ist, baut Open Science auf einem democratic turn und neuen technologischen Möglichkeiten durch die Digitalisierung auf.

- Herausforderung und Ziel: Beide Konzepte zielen auf eine Transformation des Wissenssystems, die das Verhältnis von Forschung und Gesellschaft adressiert und - auf unterschiedliche Weise - für eine neue und stärkere Einbindung plädiert. Hier divergieren beide Ansätze jedoch in ihrer Ausrichtung, da RRI die prinzipielle Legitimität und Rechtfertigbarkeit von Forschung und Innovation sowie eine tiefgreifende mutual responsiveness und sogar Ethisierung von Forschung und Technologien impliziert. Open Science hingegen fokussiert zunächst auf eine Demokratisierung durch Zugang und Einbindung.

- Akteure: Beide Konzepte betreffen Akteure des Forschungsund Innovationssystems. Open Science beschränkt sich dabei jedoch weitgehend auf WissenschaftlerInnen und deren (un) mittelbare Adressaten, während RRI alle Akteure des Innovationssystems von ForscherInnen über Unternehmen bis hin zur Politik involviert.

- Rolle und Einbezug der Gesellschaft: Beide Konzepte adressieren die Gesellschaft, indem sie darauf abzielen, die Grenzen zwischen Forschung und Gesellschaft aufzubrechen und poröser zu gestalten. RRI strebt dies über einen tiefgreifenden institutionellen Wandel an, der alle Akteure des Innovationssystems berücksichtigt. Demgegenüber nimmt Open Science die Gesellschaft eher als Adressat in den Blick.

\section{Responsible Research and Innovation kann}

\section{Open Science in einen normativen Rahmen einfügen.}

- Ansätze und Methoden: Während beide Konzepte diverse Tools und Toolboxes entwickeln, unterscheiden sich diese im Hinblick auf Adressaten, Inhalt und Funktionsebene. RRIAnsätze umfassen eine Vielzahl an Engagementmethoden, die neben der Gesellschaft vor allem auch Stakeholder aus Industrie und Politik einbinden sollen. Open-Science-Methoden legen einen anderen Schwerpunkt, insofern sie auf Informationen, Forschungsdaten und -ergebnisse sowie technische Infrastrukturen fokussieren.

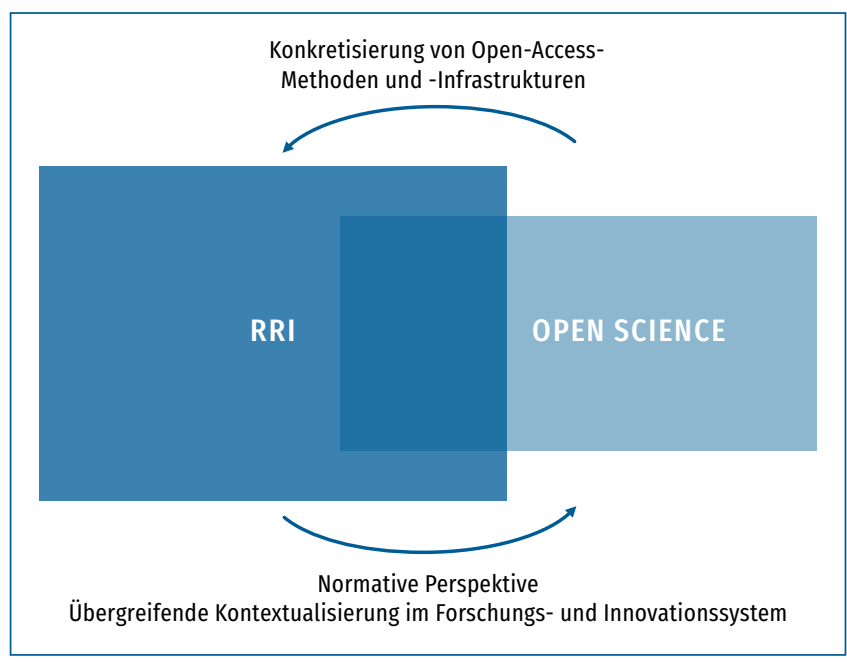

Abb. 1: Schnittmengen und Lernpotenziale zwischen RRI und Open Science. Quelle: Eigene Darstellung

Vor dem Hintergrund dieser Gegenüberstellung wird im Folgenden vorgeschlagen, dass RRI einen substantiellen Rahmen für eine konzeptuelle Entwicklung von Open Science bieten kann: Indem RRI das grundlegende Verhältnis von Wissenschaft und Gesellschaft thematisiert, kann es die unterschiedlichen Fäden und Aspekte von Open Science (stärkere gesellschaftliche Partizipation, demokratische Zugänglichkeit zu Forschungsergebnissen, weitere interdisziplinäre Zusammenarbeit, funktionierende Infrastruktur und eine sich ändernde Evaluation von Wissenschaft) bündeln und in einen normativen Rahmen einfügen. Transdisziplinäre Partizipation, der öffentliche Zugang zu Forschungsergebnissen oder die Entwicklung und der Einsatz von neuen Tools und Plattformen können damit Instanzen eines RRIgeleiteten institutionellen Wandels darstellen. Dies betrifft nicht zuletzt die grundsätzliche Frage danach, für wen Wissenschaft geöffnet werden soll und wen eine Öffnung letztlich überhaupt erreicht. Hier macht RRI darauf aufmerksam, dass eine Adressierung aller auch geschlechtsspezifische Hürden und mögliche Diskriminierungen von Minderheiten berücksichtigen muss. AuBerdem ermöglicht RRI, ethische und soziale Implikationen von Open Science frühzeitig zu bedenken und in Forschungsprozesse zu integrieren. Gleichzeitig kann RRI von Open Science lernen, wie ein inhaltlich breiteres Verständnis von Open Access konkretisiert und in (IT-)Infrastrukturen umgesetzt werden kann. Dies verdeutlicht, inwiefern beide Konzepte voneinander lernen und profitieren können: Während Open Science an Dichte gewinnen und inhaltlich präzisiert werden kann, wird für RRI eine Erweiterung um die Open-Access-Säule erreicht. Abbildung 1 stellt die Schnittmengen, Unterschiede und Lernpotenziale von RRI und Open Science schematisch dar.

Die hier vorgeschlagene Einbettung von Open Science in RRI führt zu einer Reevaluierung der Ansprüche, Implikationen und Konsequenzen von Open Science im Hinblick auf die grundlegende Öffnung von Forschung mit der und für die Gesellschaft. Damit erreicht sie die Einbindung der mitunter eher 
technisch geprägten Perspektiven und Ansätze von Open Science in forschungspolitische und gesellschaftlich-normative $\mathrm{Zu}$ sammenhänge: In einem RRI-geleiteten Verständnis kann Open Science als dynamische Öffnung für diverse gesellschaftliche Perspektiven und als Teil verantwortlicher Forschung mittels unterschiedlicher Methoden und neuer Technologien konzeptualisiert werden.

\section{Ausblick}

Das vorliegende Plädoyer für ein RRI-geleitetes Verständnis von Open Science zielt keineswegs darauf ab, den lebhaften und produktiven Diskurs differierender Konzeptualisierungen zu unterbrechen oder gar zu beschließen, vielmehr wird vorgeschlagen, die Schnittmengen und Distinktionen beider Metakonzepte für die Weiterentwicklung beider nutzbar zu machen. Dadurch kann sich sowohl für Open Science als auch für RRI ein signifikanter Mehrwert ergeben. Weitere Untersuchungen dieses Potenzials können dabei sowohl theoretische Implikationen in den Blick nehmen als auch auf praktischer Ebene Methoden diskutieren. Dabei scheinen verschiedene Perspektiven und Stoßrichtungen denkbar: Kann eine konzeptuelle Integration von Open Science in RRI als offenes Rahmenkonstrukt argumentiert und produktiv gemacht werden? Oder sollten die Distinktionen beider Ansätze eher betont und verstärkt werden, um die unterschiedlichen Schwerpunkte beider Konzepte und Debatten besser auseinanderzuhalten? Grundsätzlich können solche Forschungsansätze dazu beitragen, beide Konzepte reflexiver zu gestalten und sowohl deren Praktiken und theoretische Debatten als auch entsprechende politische Maßnahmen und Förderrichtlinien zu schärfen.

\section{Danksagung}

Durch die hilfreichen Kommentare der beiden Reviewer konnte der Artikel erheblich verbessert werden.

\section{Literatur}

Barben, Daniel; Fisher, Erik; Selin, Cynthia; Guston, David H. (2007): Anticipatory Governance of Nanotechnology: Foresight, Engagement, and Integration. In: Edward J. Hackett, Olga Amsterdamska und Judy Wajcman (Hg.): The Handbook of Science and Technology Studies. Cambridge, MA: MIT Press, S. $979-1000$

Bartling, Sönke; Friesike, Sascha (2014): Towards Another Scientific Revolution. In: Sönke Bartling und Sascha Friesike (Hg.): Opening Science. Cham: Springer International Publishing, S. 3-15.

Bogner, Alexander (Hg.) (2013): Ethisierung der Technik - Technisierung der Ethik. Der Ethik-Boom im Lichte der Wissenschafts- und Technikforschung. BadenBaden: Nomos.

Bogner, Alexander; Decker, Michael; Sotoudeh, Mahshid (2015): Technikfolgenabschätzung und „Responsible Innovation“. In: Alexander Bogner, Michael Decker und Mahshid Sotoudeh (Hg.): Responsible Innovation: Neue Impulse für die Technikfolgenabschätzung? Baden-Baden: Nomos (Gesellschaft Technik - Umwelt, 18), S. 11-28.
Carayannis, Elias G.; Campbell, David F. J. (2009): ,Mode 3`and ,Quadruple Helix: Toward a 21 ${ }^{\text {st }}$ Century Fractal Innovation Ecosystem. In: International Journal of Technology Management 46 (3/4), S. 201-234. DOI: 10.1504/ IJTM.2009.023374.

Carayannis, Elias G.; Campbell, David F.J. (2011): Mode 3 Knowledge Production in Quadruple Helix Innovation Systems. Twenty-first-century Democracy, Innovation, and Entrepreneurship for Development. In: SpringerBriefs in Business (7), S. 1-63. DOI: 10.1007/978-1-4614-2062-0_1.

Elam, Mark; Bertilsson, Margareta (2003): Consuming, Engaging and Confronting Science. In: European Journal of Social Theory 6 (2), S. 233-251. DOI: $10.1177 / 1368431003006002005$.

Etzkowitz, Henry; Leydesdorff, Loet (2000): The Dynamics of Innovation: From National Systems and "Mode 2" to a Triple Helix of University - Industry Government Relations. In: Research Policy 29 (2), S. 109-123. DOI: 10.1016/ S0048-7333(99)00055-4

European Commission (2012): Responsible Research and Innovation. Europe's Ability to Respond to Societal Challenges. Brussels (Research and Innovation). Online verfügbar unter https://ec.europa.eu/research/swafs/pdf/pub_ public_engagement/responsible-research-and-innovation-leaflet_en.pdf, zuletzt geprüft am 09.06.2017.

European Commission (2013): Responsible Research and Innovation (RRI), Science and Technology. Summary. European Commission - DirectorateGeneral for Research and Innovation. Brussels (Special Eurobarmometer, 401). Online verfügbar unter http://ec.europa.eu/commfrontoffice/ publicopinion/archives/ebs/ebs_401_en.pdf, zuletzt geprüft am 09.06.2017.

European Commission (2016): Open Innovation, Open Science, Open to the World - a Vision for Europe. Directorate-General for Research and Innovation. Brussels (KI-04-16-263-EN-N). Online verfügbar unter http://www.openaccess.gr/sites/openaccess.gr/files/Openinnovation.pdf, zuletzt geprüft am 09.06.2017.

Fecher, Benedikt; Friesike, Sascha (2014): Open Science: One Term, Five Schools of Thought. In: Sönke Bartling und Sascha Friesike (Hg.): Opening Science. Cham: Springer International Publishing, S. 17-47.

Fisher, Erik; Rip, Arie (2013): Responsible Innovation: Multi-level Dynamics and Soft Intervention Practices. In: Responsible Innovation. Chichester, UK: John Wiley \& Sons, S. 165-183.

Funtowicz, Silvio 0.; Ravetz, Jerome R. (1993): The Emergence of Post-normal Science. In: René von Schomberg (Hg.): Science, Politics and Morality. Dordrecht: Springer, S. 85-123.

Gibbons, Michael (2000): Mode 2 Society and the Emergence of Context-sensitive Science. In: Science and Public Policy 27 (3), S. 159-163. DOI: 10.3152/ 147154300781782011

Gibbons, Michael; Limoges, Camille; Nowotny, Helga; Schwartzman, Simon; Scott, Peter; Trow, Martin (1994): The New Production of Knowledge. The Dynamics of Science and Research in Contemporary Societies. London: Sage Publications.

Grunwald, Armin (2014): Technology Assessment for Responsible Innovation. In: Jeroen van den Hoven, Neelke Doorn, Tsjalling Swierstra, Bert Jaap Koops und Henny Romijn (Hg.): Responsible Innovation 1: Innovative Solutions for Global Issues. Dordrecht: Springer, S. 15-31.

Hand, Eric (2010): Citizen Science. People Power. In: Nature 466 (7307), S. 685-687. DOI: $10.1038 / 466685 a$.

McCallie, Ellen et al. (2009): Many Experts, Many Audiences: Public Engagement with Science and Informal Science Education. A CAISE inquiry group 
report. Washington, D. C.: Center for the Advancement of Informal Science Education.

Moedas, Carlos (2015): Open Innovation, Open Science, Open to the World. Rede vom 22.6.2015 auf der Konferenz in Brüssel „A new start for Europe: Opening up to an ERA of Innovation“. Online verfügbar unter http://europa.eu/rapid/ press-release_SPEECH-15-5243_en.htm, zuletzt geprüft am 09.06.2017.

Nowotny, Helga; Scott, Peter; Gibbons, Michael (2003): ,Mode 2 Revisited: The New Production of Knowledge. In: Minerva 41 (3), S. 179-194. DOI: 10.1023/ A:1025505528250.

Owen, Richard; Bessant, John; Heintz, Maggy (Hg.) (2013): Responsible Innovation. Managing the Responsible Emergence of Science and Innovation in Society. Chichester, UK: John Wiley \& Sons.

Owen, Richard; Macnaghten, Phil; Stilgoe, Jack (2012): Responsible Research and Innovation: From Science in Society to Science for Society, with Society. In: Science and public policy. 39 (6), S.751-760. DOI: 10.1093/scipol/scs093.

Powell, Maria C.; Colin, Mathilde (2009): Participatory Paradoxes. Facilitating Citizen Engagement in Science and Technology From the Top-down? In: Bulletin of Science, Technology \& Society 29 (4), S. 325-342. DOI: 10.1177| 0270467609336308.

Rip, Arie (2010): De facto Governance of Nanotechnologies. In: Morag Goodwin, Bert Jaap Koops und Ronald. Leenes (Hg.): Dimensions of Technology Regulation. Nijmegen: Wolf Legal Publishers, S. 285-308.

Rip, Arie (2014): The Past and Future of RRI. In: Life sciences, society and policy 10 (1). DOI: $10.1186 /$ s40504-014-0017-4.

Rip, Arie (2016): The Clothes of the Emperor. An Essay on RRI In and Around Brussels. In: Journal of Responsible Innovation 3 (3), S.290-304. DOI: 10.1080/ 23299460.2016.1255701.

Schäfer, M. S. (2009): From Public Understanding to Public Engagement. An Empirical Assessment of Changes in Science Coverage. In: Science Communication 30 (4), S. 475-505. DOI: 10.1177/1075547008326943.

Schmidt, Birgit; Orth, Astrid; Franck, Gwen; Kuchma, Iryna; Knoth, Petr; Carvalho, José (2016): Stepping up Open Science Training for European Research.

In: Publications 4 (2), S. 16. DOI: 10.3390/publications4020016.

Schomberg, René von (2013): A Vision of Responsible Research and Innovation.
In: Richard Owen, John Bessant und Maggy Heintz (Hg.): Responsible Innovation. Managing the Responsible Emergence of Science and Innovation in Society. Chichester, UK: John Wiley \& Sons, S. 51-74.

Schomberg, René von (2014): The Quest for the 'Right' Impacts of Science and Technology. A Framework for Responsible Research and Innovation. In: Jeroen van den Hoven, Neelke Doorn, Tsjalling Swierstra, Bert Jaap Koops und Henny Romijn (Hg.): Responsible Innovation 1: Innovative Solutions for Global Issues. Dordrecht: Springer, S. 33-50.

Siune, Karen et al. (2009): Challenging Futures of Science in Society. Emerging Trends and Cutting-edge Issues. Report of the MASIS expert group setup by the European Commission. Brussels. Online verfügbar unter http://www.itas. kit.edu/pub/v/2009/siua09a.pdf, zuletzt geprüft am 09.06.2017.

Stilgoe, Jack; Owen, Richard; Macnaghten, Phil (2013): Developing a Framework for Responsible Innovation. In: Research Policy 42 (9), S. 1568-1580. DOI: 10.1016/j.respol.2013.05.008

Tancoigne, Elise; Randles, Sally; Joly, Pierre-Benoît (2016): Evolution of a Concept: A Scientometric Analysis of RRI. In: Ralf Lindner et al. (Hg.): Navigating Towards Shared Responsibility in Research and Innovation. Approach, Process and Results of the Res-AGorA Project. Karlsruhe: Fraunhofer Institute for Systems and Innovation Research ISI, S. 39-44.

\section{JOHANN JAKOB HÄUSSERMANN}

arbeitet am Fraunhofer Center for Responsible Research and Innovation am IAO und beendet derzeit sein Masterstudium in Philosophie, Politik- und Rechtswissenschaften an der Freien Universität Berlin mit einer Arbeit im Bereich Philosophy \& Economics zu ethischen Implikationen verhaltensökonomischer Ansätze und Instrumente.

\section{MARIE HEIDINGSFELDER}

ist wissenschaftliche Mitarbeiterin am Fraunhofer Center for Responsible Research and Innovation am IAO. Nach einem Studium in Medien- und Kommunikationswissenschaften promoviert sie aktuell an der UdK Berlin. Ihr Forschungsfokus liegt auf neuen Formaten für partizipative Innovationsprozesse und neuen Ansätzen im Wissenstransfer.

\section{GAIA Masters Student Paper Award}

\section{Have your work awarded and published in a renowned scientific journal!}

The international journal GAIA - Ecological Perspectives for Science and Society invites liasters students to participate in the 2018 GAIA Miasters Student Paper Award.

\section{Submission guidelines and more information:}

WWw.oekom.de/zeitschriften/gaia/student-paper-award

Deadine: Hovember 6,2017. Anticipated publication date: June 2018.

The winner will be granted a prize money of EUR 1,500 endowed

by the Selbach Umwelt Stiftung, as well as a free one-year Selbach Umwelt Stiftung subscription to GAIA, including free online access. 